\title{
Diversidade de Pentatomoidea (Hemiptera, Heteroptera) em três fragmentos de Mata Atlântica no sul de Santa Catarina
}

\author{
Luiz A. Campos, Tanise B. P. Bertolin, Renato A. Teixeira \& Fernanda S. Martins
}

Laboratório para o Estudo de Hemípteros, Universidade do Extremo Sul Catarinense, Av. Universitária 1105, Caixa Postal 3167, 88806000 Criciúma SC, Brasil. (lac@unesc.net)

\begin{abstract}
Diversity of Pentatomoidea (Hemiptera, Heteroptera) in three fragments of Atlantic Forest in southern Santa Catarina, Brazil. The composition and the seasonal variation of Pentatomoidea (Hemiptera) were evaluated between September 2005 and August 2006 in three fragments of Atlantic Forest in the southern region of the State of Santa Catarina (Brazil): Parque

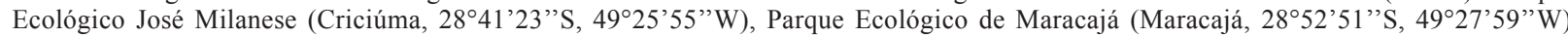
and Balneário Morro dos Conventos (Araranguá, $28^{\circ} 56^{\prime} 05^{\prime \prime} \mathrm{S}, 49^{\circ} 21^{\prime} 47^{\prime \prime} \mathrm{W}$ ). Monthly sampling had been carried out in the three areas, using beating tray and sweeping nets to sample forest edge vegetation. After an effort of 108 hours, 595 individuals of 4 families, 29 genera and 49 species. were collected. Pentatomidae was the most abundant family (82.69\%), followed by Cydnidae (15.97\%), Scutelleridae $(0.84 \%)$ and Tessaratomidae $(0.50 \%)$. Pentatomidae also presented the greatest richness with 37 species. The most abundant species were Mormidea notulifera Stål, 1860, Oebalus ypsilongriseus (De Geer, 1773), Arvelius albopunctatus (De Geer, 1773), Edessa subrastrata Bergroth, 1891, Galgupha schulzii (Fabricius, 1781) and Agroecus scabricornis (Herrich-Schäffer, 1844). More individuals were captured between the end of the spring and the beginning of autumn, representing $71.76 \%$ of the total collected. Parque do Maracaja presented significantly higher abundance and richness. This study portrays the first inventory of the diversity of Pentatomoidea in natural habitats in the State of Santa Catarina.
\end{abstract}

KEYWORDS. Stink bugs, biodiversity, community ecology, Atlantic Forest.

RESUMO. A composição e a variação sazonal da fauna de Pentatomoidea (Hemiptera) foi avaliada entre setembro de 2005 e agosto de 2006 em três fragmentos de Mata Atlântica na região sul de Santa Catarina (Brasil): Parque Ecológico José Milanese (Criciúma,

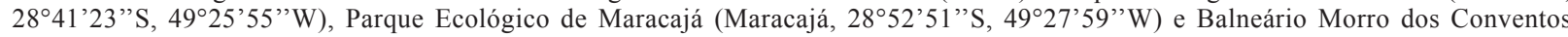
(Araranguá, $28^{\circ} 56^{\prime} 05^{\prime} \mathrm{S}, 49^{\circ} 21^{\prime} 47^{\prime} \mathrm{W}$ ). Foram realizadas coletas mensais ao longo de trilhas nas três áreas, utilizando guarda-chuva entomológico e rede de varredura para amostrar nas bordas de mata. Para um esforço amostral de 108 horas foram coletados 595 indivíduos, distribuídos em 4 famílias, 29 gêneros e 49 espécies. Pentatomidae foi a família mais abundante (82,69\%) seguida de Cydnidae $(15,97 \%)$, Scutelleridae $(0,84 \%)$ e Tessaratomidae $(0,50 \%)$. Pentatomidae também apresentou a maior riqueza com 37 espécies. As espécies mais abundantes foram Mormidea notulifera Stål, 1860, Oebalus ypsilongriseus (De Geer, 1773), Arvelius albopunctatus (De Geer, 1773), Edessa subrastrata Bergroth, 1891, Galgupha schulzii (Fabricius, 1781) e Agroecus scabricornis (Herrich-Schäffer, 1844). O período de maior captura foi entre o final da primavera e início do outono, representando $71,76 \%$ do total coletado. O Parque do Maracajá apresentou abundância e riqueza sgnificativamente maiores do que as demais áreas. Este estudo representa o primeiro inventário da diversidade de Pentatomoidea em habitats naturais no estado de Santa Catarina.

PALAVRAS-CHAVE. Percevejos-do-mato, biodiversidade, ecologia de comunidades, Mata Atlântica.

A Mata Atlântica é considerada um dos mais ricos conjuntos de ecossistemas em termos de diversidade biológica do planeta (CAPOBIANCO, 2002). Distribuída ao longo de mais de 23 graus de latitude sul é composta de uma série de fitofisionomias bastante diversificadas, apresentando uma significativa diversificação ambiental e um complexo biótico de natureza vegetal e animal altamente diverso (CAPOBIANCO, 2002). Esta floresta está reduzida a menos de $8 \%$ de sua cobertura original, resultando em alterações severas para os ecossistemas que a compõem, especialmente pela alta fragmentação de habitat e perda de sua biodiversidade (RAMBALDI \& Oliveira, 2003).

Apesar da devastação, a Mata Atlântica ainda contém uma parcela significativa da diversidade biológica, apresentando altos níveis de endemismo (Myers et al., 2000). A conservação desta biodiversidade se faz necessária e representa um dos maiores desafios deste início de século (Viana \& Pinheiro, 1998), sendo imprescindível a realização de estudos visando seu conhecimento para implementação de medidas adequadas de conservação (Moreno, 2001). Levantamentos faunísticos são ferramentas básicas para tais estudos e para o monitoramento ao longo do tempo, seja perante condições ambientais distintas, seja em resposta aos impactos de processos naturais ou de atividades humanas (LEwINSOHN et al., 2001).

Os insetos têm sido alvo de inventários e estudos faunísticos com o objetivo de encontrar subsídios para que não apenas se conheça a sua diversidade, mas também sirvam de apoio para avaliação de condições ambientais (HuMPhREY et al., 1999). A diversidade e a sensibilidade a variações no ambiente tornam este grupo especialmente importante para a conservação (BROWN JR., 1997); os levantamentos de insetos fitófagos, em particular, contribuem para o reconhecimento de um segmento importante e diversificado das biotas locais e regionais (LEWINSOHN et al., 2001).

Os pentatomóideos (Hemiptera, Pentatomoidea) são insetos exclusivamente terrestres e em geral fitófagos, com a exceção das espécies predadoras da subfamília Asopinae (Pentatomidae). Schuh \& Slater (1995) 
relacionam nove famílias com ocorrência na região Neotropical: Acanthosomatidae, Canopidae, Cydnidae, Dinidoridae, Megarididae, Pentatomidae, Phloeidae, Scutelleridae e Tessaratomidae. No mundo são conhecidas mais de 5.720 espécies nominais da superfamília, sendo que mais de 600 destas ocorrem no Brasil (Grazia et al., 1999). A maior família, Pentatomidae, apresenta diversas espécies pragas de plantas cultivadas (PANIZZI et al., 2000).

São poucos os inventários faunísticos disponíveis na literatura que incluem famílias de Pentatomoidea em ecossistemas naturais. Fora do Brasil, na região Neotropical destacam-se as listas de Grazia \& BECKER (1977) para pentatomóideos coletados na Guiana Francesa, de Brailovsky (1987) para espécies de Pentatomidae na Estação Biológica Los Tuxtlas, México e de Perez-Gelabert \& Thomas (2005) para pentatomídeos na ilha de Hispaniola, República Dominicana.

No Brasil, Buckup (1961) elaborou lista de pentatomídeos para o Estado do Rio Grande do Sul a partir de informações de literatura e coleções entomológicas. GASTAL et al. (1981) caracterizaram a riqueza de pentatomídeos na grande Porto Alegre (RS) capturados com o uso de armadilha luminosa. Link \& Grazia (1983) listaram 30 espécies de Pentatomidae coletadas em armadilha luminosa na região de Santa Maria (RS). LINK \& GRAZIA (1987) relacionaram 77 espécies de pentatomídeos para a região central do Rio Grande do Sul, incluindo informações da associação entre percevejos e plantas hospedeiras. Costa et al. (1995) identificaram, em São Sepé (RS), dez espécies de Pentatomidae sobre sete espécies vegetais. PaUla \& Ferreira (1998) realizaram um levantamento de heterópteros utilizando armadilha luminosa em mata ciliar em Viçosa (MG), registrando Pentatomidae e Cydnidae. GrAZIA et al. (1999) elaboraram lista de pentatomóideos para o Estado de São Paulo a partir de dados de literatura e material identificado pela primeira autora. BARCELLOS (2006) caracterizou a comunidade de Hemiptera em áreas de restinga junto à laguna dos Patos (RS), registrando Pentatomidae como a família com maior diversidade. Schmidt \& Barcellos (2007) analisaram padrões de riqueza e abundância da comunidade de Pentatomoidea em duas trilhas no Parque Estadual do Turvo (RS), identificando 56 espécies pertencentes às famílias Pentatomidae, Cydnidae, Scutelleridae, Cyrtocoridae, Dinidoridae, Megarididae e Tessaratomidae. Para o Estado de Santa Catarina e para a Mata Attântica não há estudos publicados acerca da comunidade de Pentatomoidea, sendo que os poucos dados existentes sobre o grupo são oriundos de trabalhos taxonômicos ou de ocorrência de espécies de interesse agrícola, estando dispersos na literatura especializada.

O objetivo deste estudo foi descrever a comunidade de Pentatomoidea em três fragmentos de Mata Atlântica no sul de Santa Catarina, avaliando composição, abundância e riqueza de espécies, bem como a similaridade entre as áreas e a variação sazonal, contribuindo assim com informações que poderão servir como subsídio para o monitoramento e a conservação de fragmentos florestais na região.

\section{MATERIAL E MÉTODOS}

Áreas de estudo. O presente estudo foi desenvolvido em três fragmentos florestais nos municípios de Criciúma, Maracajá e Araranguá, sul do Estado de Santa Catarina (Fig. 1). Os fragmentos encontram-se inseridos no Bioma Mata Atlântica, apresentam características de Floresta Ombrófila Densa e clima do tipo Cfa segundo o sistema de classificação de Köeppen, subtropical úmido com verões quentes e sem estação seca definida (OMETTO, 1981). O Parque Ecológico José Milanese (JM, 28 $41^{\prime} 23^{\prime}$ 'S, 49 $9^{\circ} 25^{\prime} 55^{\prime}$ 'W) está localizado na zona urbana do município de Criciúma, apresentando cerca de 7 ha de Floresta Ombrófila Densa Submontana. O Parque Ecológico de Maracajá (PM, $28^{\circ} 52^{\prime} 51^{\prime} \mathrm{S}, 49^{\circ} 27^{\prime} 59^{\prime}$ 'W) localiza-se no município homônimo e apresenta cerca de 110 ha de vegetação, com predominância de Floresta Ombrófila Densa de Terras Baixas, estando circundado por plantações de arroz e limitado a sudeste pela rodovia BR 101. O Balneário Morro dos Conventos (MC, 28 $56^{\circ} 05^{\prime \prime} \mathrm{S}, 49^{\circ} 21^{\prime} 47^{\prime \prime} \mathrm{W}$ ) localiza-se em Araranguá, entre a foz do rio Araranguá e o Balneário Arroio do Silva, ocupando cerca de $7 \mathrm{~km}^{2}$ de costa, sendo que o local de estudo representa um encrave de aproximadamente 25 ha de Floresta Ombrófila Densa Submontana sobre uma paleofalésia a $80 \mathrm{~m}$ de altitude, estando circundado por vegetação de restinga juntamente $\mathrm{a}$ áreas urbanizadas.

Amostragem. Cada fragmento foi amostrado uma vez por mês entre setembro de 2005 e agosto de 2006, no período das $9 \mathrm{~h}$ às $12 \mathrm{~h}$. As amostragens foram realizadas ao longo de trilhas nas bordas de mata, perfazendo duas horas de coleta utilizando-se guarda-chuva entomológico e uma hora com rede de varredura, totalizando $108 \mathrm{~h}$ de amostragem. Utilizou-se um guarda-chuva entomológico de $1 \mathrm{~m}^{2}$ para amostrar a vegetação arbustivo-arbórea com ramos entre $0,5 \mathrm{~m} \mathrm{e} 2 \mathrm{~m}$ de altura do solo, sendo a primeira árvore da trilha escolhida e as demais selecionadas por sorteio com o uso de um dado; o número obtido com o lançamento do dado determinava a próxima árvore amostrada ao longo da trilha, entre a primeira e a sexta, e

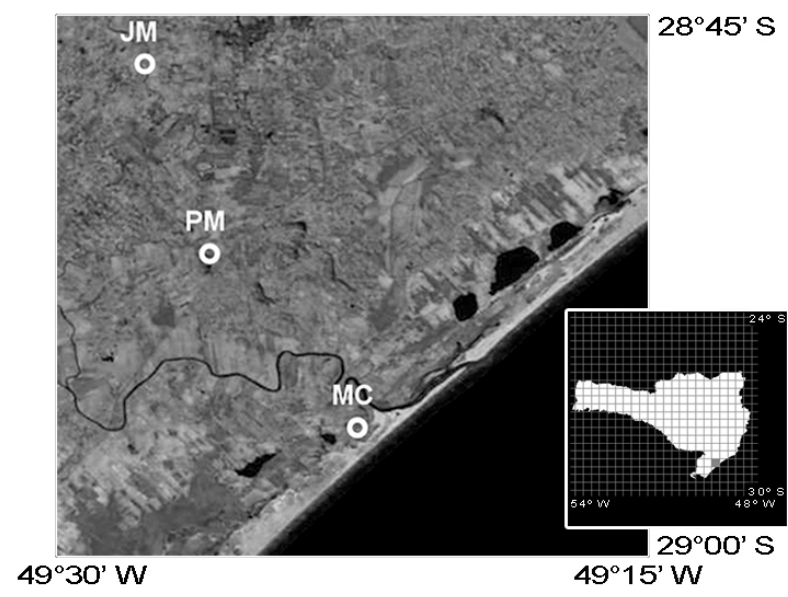

Figura 1. Localização e imagem de satélite dos fragmentos florestais amostrados no sul de Santa Catarina, Brasil (JM, Parque Ecológico José Milanese; MC, Balneário Morro dos Conventos; PM, Parque Ecológico de Maracajá). Escala 1:50.000. Fonte: Miranda \& CoutinHo (2004) 
assim sucessivamente. Cada amostra foi obtida através de cinco batidas nos ramos sobre o guarda-chuva aberto.

A rede entomológica de varredura media $40 \mathrm{~cm}$ de diâmetro e foi utilizada para amostrar a vegetação herbácea e subarbustiva; cada amostra foi obtida através de golpes com a rede na vegetação em movimentos de avanço contido a cada 20 passos.

Identificação do material e análise dos dados. Devido à dificuldade de identificação dos imaturos apenas os indivíduos adultos foram considerados para o estudo e as identificações foram feitas com o auxílio de chaves dicotômicas (McAtee \& Malloch, 1933; RucKes, 1956; Grazia, 1978; Rolston, 1978; Rolston et al., 1980; ThOMAS \& Yonke, 1990; BArcellos \& Grazia, 2003) ou consulta a taxonomistas. As espécies não identificadas foram codificadas por números. A classificação adotada para a superfamília Pentatomoidea seguiu SchUH \& SlATER (1995) e os exemplares foram depositados na Coleção Entomológica de Referência da Universidade do Extremo Sul Catarinense (CERSC).

A composição da comunidade de Pentatomoidea amostrada nos três fragmentos foi caracterizada e comparada em termos de riqueza $(\mathrm{S})$ e abundância (n), por meio de índices de diversidade, curvas de distribuição de abundância e de rarefação. Estudos que envolvam comparações de riqueza de espécies entre áreas com tamanhos amostrais diferentes necessitam da técnica de rarefação (Gotelli \& COLWELl, 2001) e, como o número real de espécies é desconhecido, os estimadores fornecem um retrato mais preciso dos padrões de riqueza (COLWELL et al., 2004).

Para a análise da distribuição de abundâncias foram consideradas espécies raras ou pouco frequentes aquelas com menos de $1 \%$ de abundância relativa e dominantes aquelas com abundância relativa acima de 10\%. Foram calculados os índices de equitabilidade (J) e de diversidade de Shannon $(\mathrm{H})$ para caracterizar respectivamente a uniformidade e a diversidade em cada área (MAGURRAN, 2004). Estas análises foram realizadas com o uso do programa PASt 1.18 (HAMMER et al., 2001). A comparação da diversidade encontrada nas áreas também foi realizada por meio dos índices de similaridade de Jaccard e de Bray-Curtis, calculados com o programa EstimateS 8.0 (Colwell, 2005). Utilizou-se o mesmo programa para o cálculo dos estimadores de riqueza Jacknife de primeira ordem, Chao 1, Bootstrap e MichaelisMenten, os quais foram selecionados por mostrarem-se menos sensíveis a variações no tamanho das amostras (HorTal et al., 2006).

\section{RESULTADOS}

Em 108 h de coleta obteve-se um total de 595 indivíduos de 49 espécies de Pentatomoidea, distribuídas em 29 gêneros de quatro famílias (Tab. I), sendo que Pentatomidae apresentou maiores abundância (n) e riqueza (S), com 492 indivíduos de 37 espécies, seguida de Cydnidae, Scutelleridae e Tessaratomidae. Foram registradas 18 espécies representadas por um indivíduo (singletons) e duas por dois (doubletons), equivalendo a $40 \%$ do total de espécies encontrado.
As espécies mais abundantes foram Mormidea notulifera Stål, 1860 (16,8\%), Oebalus ypsilongriseus (De Geer, 1773) (10,8\%) e Arvelius albopunctatus (De Geer, $1773)(8,6 \%)$. Considerando cada área isoladamente, o Parque Ecológico de Maracajá (PM) apresentou maiores abundância e riqueza $(\mathrm{n}=311 ; \mathrm{S}=33 ; \mathrm{H}=2,73)$, o Balneário Morro dos Conventos (MC) foi a segunda área em termos de riqueza e a terceira em abundância $(\mathrm{n}=102 ; \mathrm{S}=27 ; \mathrm{H}$ $=2,77$ ), situação que se inverteu no Parque Ecológico José Milanese $(J M)(n=182 ; \mathrm{S}=25 ; \mathrm{H}=2,59)$.

Conforme os resultados dos estimadores de riqueza Bootstrap e Michaelis-Menten, entre 71\% e 85\% da fauna local de Pentatomoidea foram registrados no PM, entre $50 \%$ e $86 \%$ no JM e entre $37 \%$ e $81 \%$ no MC. Dentre as espécies compartilhadas, 14 (28\%) foram registradas nos três fragmentos, indicando uma pequena similaridade entre as áreas apesar da proximidade geográfica, sendo que 26 espécies (53\%) foram exclusivas de apenas uma das áreas (Tab. I). Porém, quando são consideradas apenas as espécies com mais de dois indivíduos em pelo menos uma das áreas, este número cai para oito espécies exclusivas, sendo cinco no PM, uma no JM e duas no MC. A similaridade entre pares de áreas variou entre $16 \mathrm{e}$ 17 espécies, resultando em valores relativamente elevados e próximos do índice de Jaccard; o índice de Bray-Curtis, entretanto, indicou maior similaridade entre PM e JM e menor entre PM e MC (Tab. II). As curvas de rarefação calculadas para uma subamostra de 101 indivíduos indicaram diferenças entre $\mathrm{MC}\left(\mathrm{n}^{\circ}\right.$ estimado de espécies $=26,87 ; \mathrm{dp}=0,33)$ e JM $(20,53 \pm 1,59)$ e entre MC e PM (21,66 $\pm 2,09)$ (Fig. 2).

A proporção de espécies dominantes diferiu em cada local, resultando em menor equitabilidade no PM (J $=0,78)$, seguido do JM $(\mathrm{J}=0,80)$ e do $\mathrm{MC}(\mathrm{J}=0,84)$. A distribuição das abundâncias resultou em predomínio de espécies intermediárias no JM (48\%) e no MC (50\%), enquanto que no PM a maior proporção foi de espécies raras (64\%) (Fig. 3). No PM as espécies dominantes foram M. notulifera (15\%), A. albopunctatus (14\%), Edessa subrastrata Bergroth, 1891 (13\%) e O. ypsilongriseus (10\%); no JM, M. notulifera (24\%) e O. ypsilongriseus (14\%) e no MC, Thyanta humilis Bergroth, 1891 (17\%) e Piezodorus guildinii (Westwood, 1837) (16\%). Com

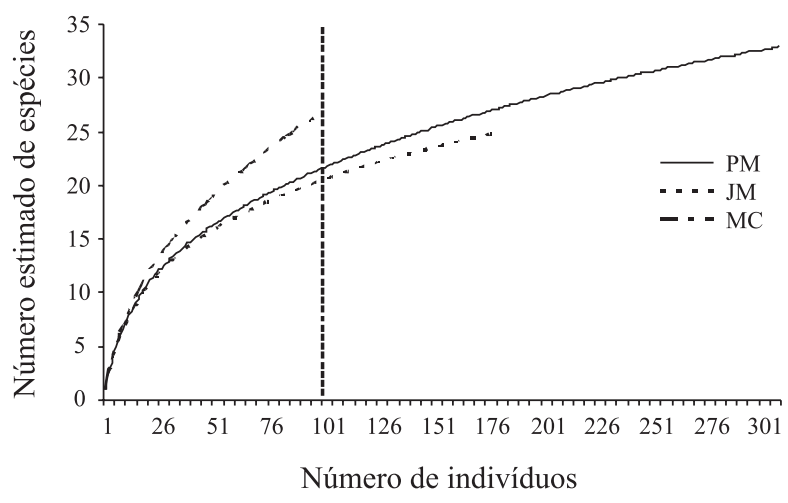

Figura 2. Curvas de rarefação para a riqueza estimada de espécies de Pentatomoidea em três fragmentos florestais no sul de Santa Catarina, Brasil, setembro de 2005 a agosto de 2006 (JM, Parque Ecológico José Milanese; MC, Balneário Morro dos Conventos; PM, Parque Ecológico de Maracajá). 
relação às espécies raras, no PM foram registradas 11 singletons e cinco doubletons, no JM oito singletons e três doubletons, e no MC 13 singletons e cinco doubletons.

A distribuição da diversidade ao longo do período amostral resultou em menores valores de abundância e riqueza no inverno, enquanto que os maiores picos de abundância ocorreram em dezembro de 2005 no PM, em março de 2006 no JM e em abril de 2006 no MC. Os picos de riqueza acompanharam os de abundância, com a exceção do PM onde a maior riqueza foi registrada em novembro de 2005 (Fig. 4). O período de maior captura de Pentatomoidea ocorreu entre o final da primavera e o início do outono, quando foram registrados 427 indivíduos $(71,76 \%)$.

Tabela I. Espécies de Pentatomoidea registradas em três fragmentos florestais no sul de Santa Catarina, Brasil, setembro de 2005 a agosto de 2006 (JM, Parque Ecológico José Milanese; MC, Balneário Morro dos Conventos; n, número de indivíduos; PM, Parque Ecológico de Maracajá).

\begin{tabular}{|c|c|c|c|c|}
\hline \multirow[t]{2}{*}{ Taxons } & \multicolumn{3}{|c|}{$\mathrm{n}$} & \multirow[b]{2}{*}{ Total } \\
\hline & $\mathrm{PM}$ & $\mathrm{JM}$ & $\mathrm{MC}$ & \\
\hline \multicolumn{5}{|l|}{ Pentatomidae } \\
\hline \multicolumn{5}{|l|}{ Asopinae } \\
\hline Podisus crassimargo (Stål, 1860) & 1 & & & 1 \\
\hline P. distinctus (Stål, 1860) & 14 & & & 14 \\
\hline P. ventralis (Dallas, 1851) & & & 1 & 1 \\
\hline P. nigrispinus (Dallas, 1851) & 2 & & 2 & 4 \\
\hline Tynacantha marginata Dallas, 1851 & 8 & 1 & 1 & 10 \\
\hline \multicolumn{5}{|l|}{ Edessinae } \\
\hline Brachystethus geniculatus (Fabricius, 1787) & 1 & & & 1 \\
\hline Edessa impura Bergroth, 1891 & & 1 & & 1 \\
\hline E. lineata Westwood, 1837 & 1 & & & 1 \\
\hline E. meditabunda (Fabricius, 1794) & 1 & 3 & & 4 \\
\hline E. polita (Lepeletier \& Serville, 1825) & 2 & 1 & 2 & 5 \\
\hline E. subrastrata Bergroth, 1891 & 41 & 8 & & 49 \\
\hline Peromatus notatus (Burmeister, 1835) & & & 1 & 1 \\
\hline \multicolumn{5}{|l|}{ Pentatominae } \\
\hline Agroecus scabricornis (Herrich-Schäffer, 1844) & 16 & 16 & & 32 \\
\hline Arvelius albopunctatus (De Geer, 1773) & 45 & & 6 & 51 \\
\hline Banasa sulcata Thomas, 1990 & & & 3 & 3 \\
\hline Chloropepla vigens (Stål, 1860) & & & 1 & 1 \\
\hline Chinavia difficilis (Stål, 1860) & & 1 & & 1 \\
\hline C. obstinata (Stål, 1860) & & & 1 & 1 \\
\hline Dichelops phoenix Grazia, 1978 & 1 & 3 & 6 & 10 \\
\hline Euschistus aceratos Berg, 1894 & & & 4 & 4 \\
\hline E. triangulator (Herrich-Schäffer, 1842) & 3 & & & 3 \\
\hline Lopadusa quinquedentata (Spinola, 1837) & 1 & & & 1 \\
\hline Loxa viridis (Palisot de Beauvois 1805) & & 1 & 2 & 3 \\
\hline Mayrinia curvidens (Mayr, 1864) & 2 & & & 2 \\
\hline Mormidea hamulata Stål, 1860 & 1 & 3 & 2 & 6 \\
\hline M. notulifera Stål, 1860 & 47 & 44 & 9 & 100 \\
\hline M. quinqueluteum (Lichtenstein, 1796) & 7 & 17 & 1 & 25 \\
\hline M. rugosa Rolston, 1978 & 3 & & & 3 \\
\hline Myota aerea (Herrich-Schäffer, 1841) & 1 & & & 1 \\
\hline Oebalus poecilus (Dallas, 1851) & 18 & 4 & 1 & 23 \\
\hline O. ypsilongriseus (De Geer, 1773) & 30 & 25 & 9 & 64 \\
\hline Pallantia macula (Dallas, 1851) & 2 & & & 2 \\
\hline Piezodorus guildinii (Westwood, 1837) & & 2 & 16 & 18 \\
\hline Proxys albopunctulatus (Palisot de Beauvois, 1805) & 1 & 2 & 1 & 4 \\
\hline Stictochilus tripunctatus Bergroth, 1918 & 1 & & & 1 \\
\hline Thoreyella sp. 1 & 13 & & & 13 \\
\hline Thyanta humilis Bergroth, 1891 & 6 & 5 & 17 & 28 \\
\hline \multicolumn{5}{|l|}{ Cydnidae } \\
\hline \multicolumn{5}{|l|}{ Corimelaeninae } \\
\hline Galgupha corvina (Horvath, 1919) & 3 & 10 & 1 & 14 \\
\hline G. cruralis (Stål, 1862) & 4 & 11 & 1 & 16 \\
\hline G. neobisignata McAtee \& Malloch, 1933 & 2 & 11 & 1 & 14 \\
\hline G. cf. punctifer McAtee \& Malloch, 1933 & & & 1 & 1 \\
\hline G. cf. reinhardti (Jensen-Haarup, 1926) & & 1 & & 1 \\
\hline G. schulzii (Fabricius, 1781) & 29 & 8 & 9 & 46 \\
\hline Galgupha sp. 1 & & 1 & & 1 \\
\hline Galgupha sp. 2 & & 1 & & 1 \\
\hline Pericreps sp. 1 & 1 & & & 1 \\
\hline \multicolumn{5}{|l|}{ Scutelleridae } \\
\hline Symphylus sp. 1 & & & 1 & 1 \\
\hline Orsilochides sp. 1 & & 2 & 2 & 4 \\
\hline \multicolumn{5}{|l|}{ Tessaratomidae } \\
\hline \multicolumn{5}{|l|}{ Oncomerinae } \\
\hline Piezosternum thunbergi Stål, 1860 & 3 & & & 3 \\
\hline Total & 311 & 182 & 102 & 595 \\
\hline
\end{tabular}




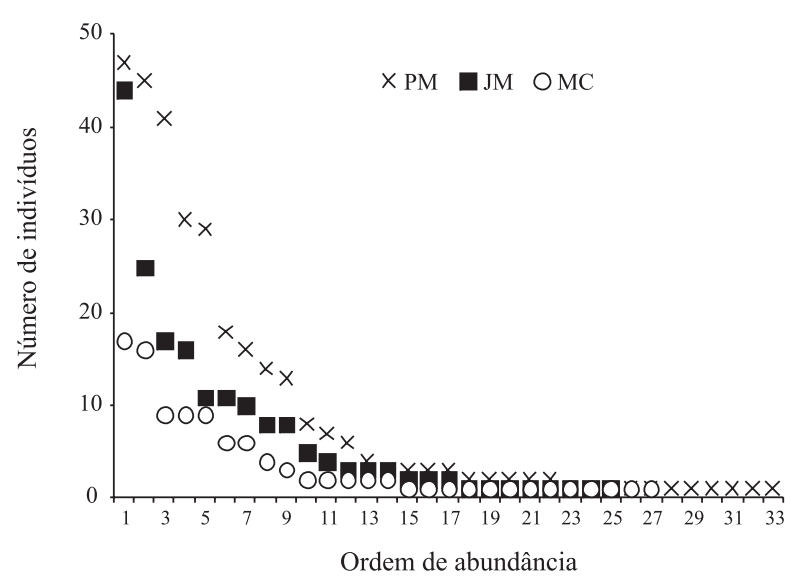

Figura 3. Distribuição da abundância das espécies de Pentatomoidea coletadas em três fragmentos florestais no sul de Santa Catarina, Brasil, setembro de 2005 a agosto de 2006 (JM, Parque Ecológico José Milanese; MC, Balneário Morro dos Conventos; PM, Parque Ecológico de Maracajá).
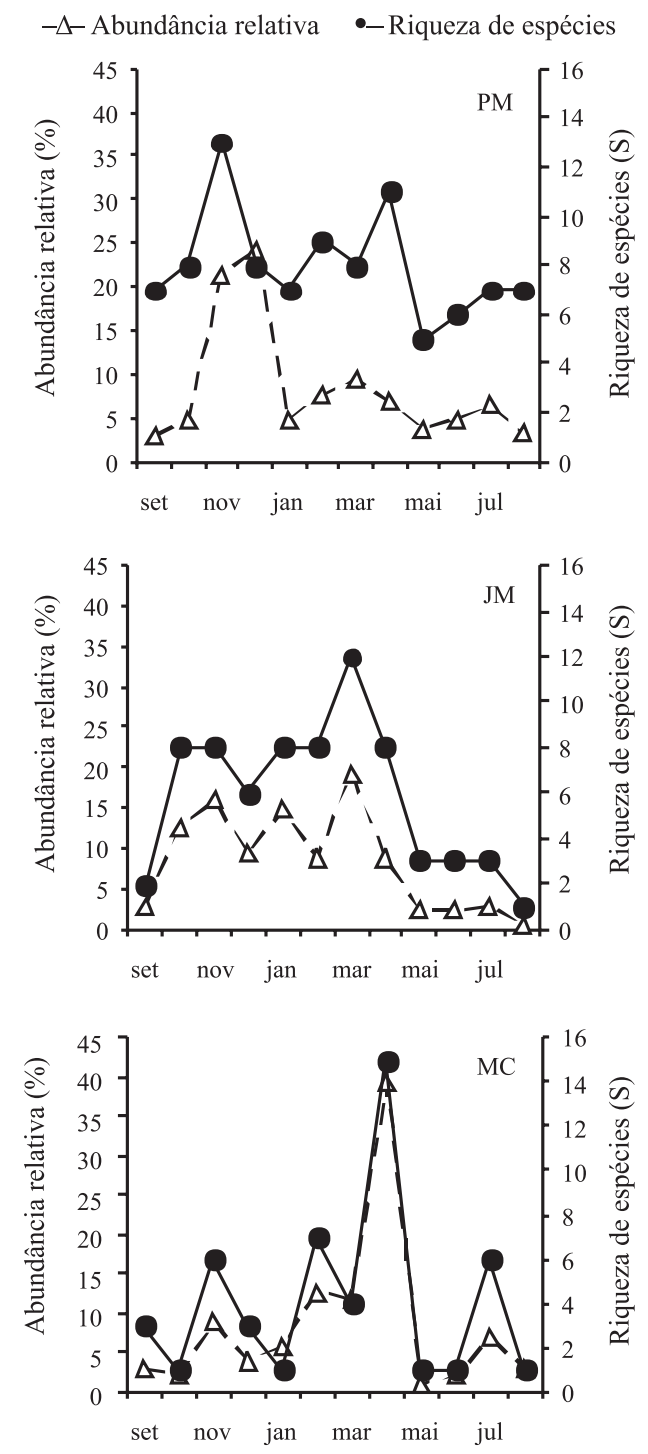

Figura 4. Abundância relativa (\%) e riqueza de espécies (S) de Pentatomoidea ao longo do período de estudo em três fragmentos florestais no sul de Santa Catarina, Brasil, setembro de 2005 a agosto de 2006 (JM, Parque Ecológico José Milanese; MC, Balneário Morro dos Conventos; PM, Parque Ecológico de Maracajá).
Tabela II. Número e proporção de espécies compartilhadas de Pentatomoidea e índices de similaridade entre os fragmentos florestais amostrados no sul de Santa Catarina no período de setembro de 2005 a agosto de 2006 (JM, Parque Ecológico José Milanese; MC, Balneário Morro dos Conventos; PM, Parque Ecológico de Maracajá; S, riqueza de espécies).

\begin{tabular}{lccccc}
\hline Área 1 x Área 2 & \multicolumn{2}{c}{ Espécies Compartilhadas } & \multicolumn{2}{c}{ Similaridade } \\
& $\begin{array}{c}\text { Total } \\
(\mathrm{S})\end{array}$ & $\begin{array}{c}\text { Área 1 } \\
(\%)\end{array}$ & $\begin{array}{c}\text { Área } 2 \\
(\%)\end{array}$ & Jaccard & Bray- \\
\hline PM x JM & 17 & 51,52 & 60,00 & 0,415 & 0,535 \\
PM x MC & 16 & 48,48 & 59,26 & 0,364 & 0,252 \\
JM x MC & 17 & 60,00 & 62,96 & 0,486 & 0,345 \\
\hline
\end{tabular}

\section{DISCUSSÃO}

A comparação dos resultados aqui apresentados pode ser feita, em certa medida, com trabalhos anteriores desenvolvidos no Rio Grande do Sul, visto serem os mais detalhados em termos de identificação das espécies e de não haver na literatura registro de levantamentos de pentatomóideos em áreas de Floresta Ombrófila Densa da Mata Atlântica.

Nos estudos realizados com armadilha luminosa por GASTAL et al. (1981) e LiNK \& GRAZIA (1983) foram registradas respectivamente 37 e 30 espécies de Pentatomidae, números considerados baixos e possivelmente relacionados às alterações ambientais dos locais de estudo (GASTAL et al., 1981) ou à pequena eficácia do método de coleta na captura de pentatomídeos (LinK \& GRAZIA, 1983). Na região central do Rio Grande do Sul, às espécies referidas por LinK \& GRAZIA (1983) foram adicionadas 47 (Link \& GRAZIA, 1987), em compilação de dados obtidos ao longo de 11 anos com o uso de diversos métodos de coleta. BArcellos (2006) amostrou hemípteros em mata de restinga arenosa e paludosa na planície costeira gaúcha, coletando 437 indivíduos de 55 espécies de Pentatomoidea. Schmidt \& Barcellos (2007), após 153 horas de esforço amostral utilizando guardachuva entomológico no Parque Estadual do Turvo, coletaram 816 indivíduos de 56 espécies de Pentatomoidea. Embora para o estado de Santa Catarina não exista uma lista de espécies de Pentatomoidea publicada, informações obtidas na literatura taxonômica e em coleções zoológicas permitem reconhecer a ocorrência no estado de cerca de 117 espécies da superfamília (Luiz A. Campos, dados não publicados).

No presente estudo, Pentatomidae foi a família mais abundante e com maior riqueza de espécies, resultado esperado visto ser esta a família mais diversa dentre os pentatomóideos. Estudos faunísticos realizados em diferentes regiões biogeográficas confirmam a tendência a uma maior coleta de Pentatomidae (KuLA \& BRYJA, 2002; Perez-Gelabert \& Thomas, 2005; Schmidt \& Barcellos, 2007). Da mesma forma os gêneros que apresentaram maior riqueza de espécies, Galgupha Amyot \& Serville, 1843, Edessa Fabricius, 1803, Mormidea Amyot \& Serville, 1843 e Podisus Herrich-Schäffer, 1851, estão entre aqueles com os maiores números de espécies nominais. SCHMIDT \& BARCELlos (2007) registraram maior riqueza de espécies para estes mesmos gêneros, juntamente com Euschistus Dallas, 1851. 
A proporção de singletons e doubletons, representando $40 \%$ do total de espécies, é esperada para comunidades de insetos herbívoros em florestas tropicais (NovotnÝ \& BASSET, 2000) e possivelmente deve-se a limitações impostas pelas metodologias de coleta adotadas, pois espécies que habitam o dossel, por exemplo, seriam coletadas apenas de forma acidental (Schmidt \& Barcellos, 2007). Outro aspecto a ser considerado é a possibilidade de que diversas espécies coletadas tenham padrões agregados de distribuição populacional, o que resultaria em desvio da distribuição das abundâncias simultaneamente em direção às espécies dominantes e em direção às raras (GREEN \& PlOTKIN, 2007). Não se descarta, porém, a possibilidade de que parte destas espécies sejam efetivamente raras, podendo representar um padrão encontrado em regiões de florestas tropicais (PRICE et al., 1995; NovotNÝ \& BASSET, 2000) ou ser resultado de processos de redução da biodiversidade em função do pequeno tamanho dos fragmentos amostrados (BierRegard JR. et al., 1992). As curvas de acumulação de espécies nos três locais não apresentaram tendência à estabilização, indicando que a ampliação do esforço amostral poderia resultar em maior número de espécies registradas.

Com relação às maiores abundâncias, dentre as espécies dominantes no PM e no JM (M. notulifera, $O$. ypsilongriseus e $A$. albopunctatus), as duas primeiras são consideradas pragas para as lavouras de arroz, trigo, aveia, entre outras (PANIZZI, 1997; PANIZZI et al., 2000) e tiveram ocorrência entre outubro de 2005 e março de 2006 , coincidindo com o cultivo de arroz na região. No MC as espécies mais abundantes foram P. guildinii e T. humilis, sendo que a primeira é uma das pragas mais importantes da lavoura de soja, além de feijão, ervilha e alfafa, sendo também encontrada em plantas hospedeiras nativas, especialmente leguminosas (PANIZZi et al., 2000). Além de pragas em potencial, estas espécies são consideradas polífagas podendo, assim, migrar de agroecossistemas adjacentes e utilizar os fragmentos florestais para abrigo e alimentação, pois espécies vegetais silvestres podem desempenhar um papel essencial no desenvolvimento de imaturos e na capacidade reprodutiva de adultos de pentatomídeos (PANIZZI, 1997).

As diferenças encontradas na diversidade de Pentatomoidea nas três áreas podem ter relação com o tamanho dos fragmentos amostrados, visto que as maiores riquezas foram registradas nos maiores fragmentos. A pequena diferença no número de espécies encontradas no JM e no MC, no entanto, indica que fatores como o estado de conservação da vegetação e o grau de pressão sobre os fragmentos não devem ser descartados; o JM é uma unidade de conservação municipal, protegida e com acesso restrito, enquanto o $\mathrm{MC}$ é uma área aberta ao trânsito de pessoas e animais domésticos. A redução na diversidade de Pentatomidae em resposta a distúrbios elevados é esperada, sendo a família um grupo eficaz para indicar impactos sobre a vegetação em áreas de Mata Atlântica (BROwN JR., 1997); os resultados aqui obtidos confirmam essa tendência e sugerem que o fragmento mais impactado seja o MC.

Este trabalho é pioneiro no estudo da diversidade de Pentatomoidea em fragmentos de Floresta Ombrófila
Densa no estado de Santa Catarina, sendo ainda o primeiro levantamento das espécies do grupo no sul do Estado. A maior captura de pentatomóideos no final da primavera, início e final de verão, bem como a não estabilização das curvas de acumulação de espécies, indicam a necessidade de se intensificar amostragens nesses períodos visando um registro mais completo da riqueza do grupo. Da mesma forma, futuros estudos em fragmentos florestais que incluam outras metodologias de amostragem deverão ampliar este registro, permitindo uma melhor caracterização da superfamília no sul catarinense.

Agradecimentos. À Dra. Jocélia Grazia (UFRGS) pela confirmação da identificação de espécies de Pentatominae e pelas críticas ao trabalho original. À Dra. Aline Barcellos Prates dos Santos (FZBRS) pela identificação de Scutelleridae e ao Dr. José Antonio Marin Fernandes (UFPA) pela identificação de Edessinae. Ao Dr. Cristiano Feldens Schwertner e ao revisor anônimo pelas valiosas sugestões.

\section{REFERÊNCIAS BIBLIOGRÁFICAS}

Barcellos, A. 2006. Hemípteros terrestres. In: Becker, F. G.; Ramos, R. A. \& Moura, L. A. orgs. Biodiversidade. Regiões da Lagoa do Casamento e dos Butiazais de Tapes, planície costeira do Rio Grande do Sul. Brasília, Ministério do Meio Ambiente. p.198-209.

Barcellos, A. \& Grazia, J. 2003. Revision of Brachystethus (Heteroptera, Pentatomidae, Edessinae). Iheringia, Série Zoologia, 93(4):413-446.

Bierregard Jr., R. O.; Lovejoy, T. E.; Kapos, V.; Santos, A. A. \& Hutchings, R. W. 1992. The Biological Dynamics of Tropical Rainforest Fragments. BioScience 42:859-866

Brailovsky, H. 1987. Hemiptera-Heteroptera de México XXXVIII. Los Pentatomini de la Estación de Biología Tropical "Luz Tuxtlas", Veracruz (Pentatomidae). Anales del Instituto de Biología, Serie Zoología, 69(1):1-154.

Brown JR., K. S. 1997. Diversity, disturbance, and sustainable use of Neotropical forests: insects as indicators for conservation monitoring. Journal of Insect Conservation 1:25-42.

Buckup, L. 1961. Os pentatomídeos do Estado do Rio Grande do Sul (Brasil) (Hemiptera-Heteroptera-Pentatomidae). Iheringia, Série Zoologia, 16:1-24.

Capobianco, J. P. R. 2002. Mata Atlântica: Conceito, abrangência e área original. In: Schäffer, W. B. \& Prochnow, M. eds. A Mata Atlântica e você. Brasília, APREMAVI. p.111-123.

Colwell, R. K. 2005. EstimateS: Statistical estimation of species richness and shared species from samples. Disponível em: <http://viceroy.eeb.uconn.edu/estimates $>$. Acesso em: 14.08.2006.

Colwell, R. K.; MaO, C. X. \& Chang, J. 2004. Interpolating, extrapolating, and comparing incidence-based species accumulation curves. Ecology 85(10):2717-2727.

Costa, E. C.; Bogorni, P. C. \& Bellomo, V. H. 1995. Percevejos coletados em copas de diferentes espécies florestais, Pentatomidae-1. Ciência Florestal. Santa Maria 5(1):123128

Gastal, H. A. De O.; Lanzer-De-Souza, M. E. \& Galileo, M. H. M. 1981. Diversidade e similaridade de comunidades de Pentatomidae (Hemiptera) capturados com armadilha luminosa na Grande Porto Alegre, Rio Grande do Sul. Iheringia, Série Zoologia, 59:5-12.

Gotelli, N. J. \& Colwell, R. K. 2001. Quantifying biodiversity: procedures and pitfalls in the measurement and comparison of species richness. Ecology Letters 4:379-391.

Grazia, J. 1978. Revisão do gênero Dichelops Spinola, 1837 (Hemiptera, Pentatomidae, Pentatomini). Iheringia, Série Zoologia, 53:3-119.

Grazia, J. \& Becker, M. 1977. The Pentatomoidea (Heteroptera) collected in French Guiana by the expedition of the Museum National d'Histoire Naturelle. Anales de la Societé Entomologique de France 13(1):53-67. 
Grazia, J.; Fortes, N. D. F. de \& Campos, L. A. 1999. Pentatomoidea. In: Brandẽo, C. R. F. \& Cancello, E. M. eds. Biodiversidade do Estado de São Paulo, Brasil: síntese do conhecimento ao final do século $\mathrm{XX}$, 5: invertebrados terrestres. São Paulo, FAPESP. p.101-112.

Green, J. L. \& Plotkin, J. B. 2007. A statistical theory for sampling species abundances. Ecology Letters 10(11):1037-1045.

Hammer, O.; Harper, D. A. T. \& Ryan, P. D. 2001. PASt: Paleontological Statistics Software Package for Education and Data Analysis. Palaeontologia Electronica 4(1):1-9. Disponível em: <http://palaeo-electronica.org/2001_1/past/ issue1_01.htm>. Acesso em: 18.11.2006.

Hortal, J.; Borges, P. A. V. \& Gaspar, C. 2006. Evaluating the performance of species richness estimators: sensitivity to sample grain size. Journal of Animal Ecology 75(1):274-287.

Humphrey, J. W.; Hawes, C.; Peace, A. J.; Ferris-Kan, R. \& Jukes, M. R. 1999. Relationships between insect diversity and habitat characteristics in plantation forest. Forest Ecology and Management 113(1):11-21.

Kula, E. \& BRYJA, J. 2002. Comparison of various sampling methods for evaluation of biodiversity of true bugs (Heteroptera) in a birch forest. Ekologia (Bratislava) 21(2):137-147.

Lewinsohn, T. M.; Prado, P. K. L. \& Almeida, A. M. 2001. Inventários Bióticos Centrados em recursos: Insetos fitófagos e plantas hospedeiras. In: Dias, B. F. S. \& Garay, I. eds. Conservação da biodiversidade em ecossistemas tropicais. Avanços conceituais e revisão de novas metodologias de avaliação e monitoramento. Petrópolis, Vozes. p.174-189.

LinK, D. \& GraZiA, J. 1983. Pentatomídeos capturados em armadilha luminosa, em Santa Maria, RS, Brasil. Anais da Sociedade Entomológica do Brasil 12(1):123-125.

1987. Pentatomídeos da região central do Rio Grande do Sul (Heteroptera). Anais da Sociedade Entomológica do Brasil 16(1):115-129.

Magurran, A. E. 2004. Measuring Biological Diversity. Oxford, Blackwell. 256p.

McAtee, W. \& Malloch, L. 1933. Revision of the subfamily Thyreocorinae of the Pentatomidae (Hemiptera-HeteropteraPentatomoidea). Annals of the Carnegie Museum 21(4):191-411.

Miranda, E. E. DE \& Coutinho, A. C. 2004. Brasil Visto do Espaço. Campinas: Embrapa Monitoramento por Satélite. Disponível em: <http://www.cdbrasil.cnpm.embrapa.br>. Acesso em: 13.11.2007.

Moreno, C. E. 2001. Métodos para medir la biodiversidad. Zaragoza, Sociedad Entomológica Aragonesa. Manuales y Tesis SEA. V.1. $84 \mathrm{p}$

Myers, N.; Mittermeier, R. A.; Mittermeier, C. G.; Fonseca, G. A. B.
DA \& KEnT, J. 2000. Biodiversity hotspots for conservation priorities. Nature 403:853-858.

Novotný, V. \& Basset, Y. 2000. Rare species in communities of tropical insect herbivores: pondering the mystery of singletons. Oikos 89(3):564-572.

Ometto, J. C. 1981. Bioclimatologia vegetal. São Paulo, Agronômica Ceres. 425p.

PAnizzi, A. R. 1997. Wild hosts of pentatomids: ecological significance and role in their pest status on crops. Annual Review of Entomology 42:99-122.

Panizzi, A. R.; McPherson, J. E.; James, D. G.; Javahery, M. \& McPherson, R. M. 2000. Stink bugs (Pentatomidae). In: Schaefer, C. W. \& Panizzi, A. C. eds. Heteroptera of economic importance. New York, CRC. p.421-474.

Paula, A. S. \& Ferreira, P. S. F. 1998. Fauna de Heteroptera de la "Mata do Córrego do Paraíso", Viçosa, Minas Gerais, Brasil. Riqueza y diversidad específicas. Anales del Instituto de Biología, Serie Zoología, 69(1):39-51.

Perez-Gelabert, D. E. \& Thomas, D. B. 2005. Stink bugs (Heteroptera: Pentatomidae) of the island of Hispaniola, with seven new species from the Dominican Republic. Boletín Sociedad Entomológica Aragonesa 37:319-352.

Price, P. W.; Diniz, I. R.; Morais, H. C. \& Marques, E. S. A. 1995. The abundance of insect herbivore species in the tropics: the high local richness of rare species. Biotropica 27(4):468-478.

Rambaldi, D. M. \& Oliveira, D. A. S. 2003. Fragmentação de Ecossistemas: Causas, efeitos sobre a biodiversidade e recomendações de políticas públicas. Brasília, MMA/SBF. 510p.

Rolston, L. H. 1978. A revision of the genus Mormidea (Heteroptera, Pentatomidae). Journal of the New York Entomological Society 86(3):161-219.

Rolston, L. H.; McDonald, F. J. D. \& Thomas, D. B, JR. 1980. A conspectus of Pentatomini genera of the Western Hemisphere - part 1 (Hemiptera: Pentatomidae). Journal of the New York Entomological Society 88(2):120-132.

Ruckes, H. 1956. New species of Thyanta Stål (Pentatomidae, Heteroptera). Bulletin of the Brooklyn Entomological Society 47(3):57-69.

Schmidt, L. S. \& Barcellos, A. 2007. Abundância e riqueza de Heteroptera (Hemiptera) do Parque Estadual do Turvo, sul do Brasil: Pentatomoidea. Iheringia, Série Zoologia, 97(1):73-79.

Schun, T. R. \& Slater, J. A. 1995. True bugs of the World (Hemiptera: Heteroptera). Classification and natural history. Ithaca, Cornell University. 336p.

Thomas, D. B., JR. \& Yonke, T. R. 1990. Review of the genus Banasa (Hemiptera: Pentatomidae) in South America. Annals of the Entomological Society of America 83(4):657-688.

Viana, V. M. \& Pinheiro, L. A. F. V. 1998. Conservação da biodiversidade em fragmentos florestais. Série Técnica IPET 12(32):25-42.

Recebido em dezembro de 2007. Aceito em outubro de 2008. ISSN 0073-4721

Artigo disponível em: www.scielo.br/isz

Iheringia, Sér. Zool., Porto Alegre, 99(2):165-171, 30 de junho de 2009 